\title{
Nasal peak inspiratory flow (NPIF) as a diagnostic tool for differentiating decongestable from structural nasal
} obstruction*

\author{
David Chin 1,2, George Marcells ${ }^{3}$, Joanne Malek4, Eleanor Pratt ${ }^{4}$, \\ Ray Sacks ${ }^{5,6}$, Kornkiat Snidvongs $s^{6,7}$, Richard Harvey ${ }^{2,6,8}$ \\ ' Department of Otolaryngology Head \& Neck Surgery, Changi General Hospital, Singapore \\ ${ }^{2}$ Department of Otolaryngology Head Neck \& Skull Base Surgery, St Vincent's Hospital, Sydney, NSW, Australia \\ ${ }^{3}$ Department of Otolaryngology, Sydney Hospital, Sydney, NSW, Australia \\ ${ }^{4}$ Faculty of Medicine, The University of Sydney, Sydney, NSW, Australia \\ ${ }^{5}$ Department of Otolaryngology, Concord General Hospital, Sydney, NSW, Australia \\ ${ }^{6}$ Australian School of Advanced Medicine, Macquarie University Hospital, Sydney, NSW, Australia \\ ${ }^{7}$ Faculty of Medicine, Chulalongkorn University, Bangkok, Thailand \\ ${ }^{8}$ Faculty of Medicine, University of New South Wales, Sydney, NSW, Australia
}

Rhinology 52: 116-121, 2014 DOI:10.4193/Rhino13.126

*Received for publication:

August 27, 2013

Accepted: December 3, 2013

\begin{abstract}
Background: Identifying the cause of nasal obstruction is critical before surgical intervention. Structural nasal obstruction, due to nasal valve stenosis, is unlikely to benefit from simple septoplasty and turbinate reduction. This study assesses changes in nasal peak inspiratory flow (NPIF) as a tool for discriminating decongestable versus structural obstruction.
\end{abstract}

Methodology: Cross-sectional study of patients undergoing nasal airflow assessment was performed. Rhinomanometry, nasal obstruction visual analogue scores (VAS) and NPIF were performed pre- and post-decongestion. Population groups were defined with decongestable or structural obstruction by relative post-decongestion changes in airways resistance and symptoms.

Results: Fifty two patients were assessed, 24 with decongestable, 28 with structural obstruction. Pre- and post-decongestion NPIF were similar between groups. Absolute and percentage NPIF change were larger with decongestable versus structural obstruction. Sensitivity and specificity for predicting decongestable obstruction were $75.0 \%$ and $60.7 \%$ for NPIF increase $>20 \mathrm{~L} / \mathrm{min} ; 75.0 \%$ and $64.3 \%$ for NPIF increase $>20 \%$. The respective positive predictive values were $62.1 \%$ and $64.3 \%$.

Conclusion: NPIF increase after decongestion is larger with decongestable than structural nasal obstruction. NPIF alone cannot discriminate the two conditions and does not replace more formal assessment.

Key words: nasal mucosa, nasal cavity, nasal surgical procedures, turbinates, nasal septum

\section{Introduction}

Nasal obstruction is a common patient complaint and has a significant impact on quality of life ${ }^{(1,2)}$. The cause of nasal obstruction may be due to inferior turbinate hypertrophy, nasal septal deformity, nasal valve stenosis or a combination of these $(3,4)$. Inferior turbinate enlargement is usually associated with venous engorgement and oedema ${ }^{(5)}$ and both the sense of nasal patency and actual airflow should improve with decongestion. When there is a lack of improvement, it is important to exclude structural obstruction secondary to nasal valve stenosis, as this will probably not respond to simple septal surgery and/ or turbinate procedures ${ }^{\left({ }^{6}\right)}$, but may necessitate a more complex approach involving correction of the various anatomical subunits constituting the nasal valve complex ${ }^{(7-9)}$. Identification of the cause of nasal obstruction is therefore critical before surgical intervention to avoid unsatisfactory outcomes.

Objective assessment of the nasal airway can provide additional information about the cause of nasal obstruction. A close relati- 
onship has been shown between patency and resistance in the nasal airway, with severe narrowing in the anterior nasal airway associated with higher nasal airway resistance (NAR) ${ }^{(10)}$. In particular, increases in NAR due to narrowing of the nasal airway are especially marked at the nasal valve region ${ }^{(11)}$.

Subjective assessments of nasal obstruction, whether patientreported or physician-based (rhinoscopy and endoscopy), do not always correlate with objective tests ${ }^{(12)}$. The relationship between subjective nasal obstruction, rhinoscopy and rhinomanometry, though significant, is weak ${ }^{(13,14)}$. Similarly, the concordance between nasal endoscopy and rhinomanometry in patients with nasal obstruction is reported to be only $66 \%{ }^{(14)}$. The assessment of changes in nasal obstruction that occur with decongestion may be very useful. The nasal decongestion test, based on changes in visual analogue scores (VAS) for nasal obstruction, is reported to be a sensitive tool for identifying increases in nasal patency after decongestion. A decrease in NAR ${ }^{(15)}$ and nasal obstruction VAS ${ }^{(16)}$ in patients with allergic rhinitis has been reported. In particular, a decrease in VAS of more than 2.65 $\mathrm{cm}$ was found to have positive predictive value (PPV) of $94.5 \%$ in predicting increased nasal airflow of $120 \mathrm{~mL} / \mathrm{s}^{(16)}$.

The relationship between NPIF and NAR with decongestion is yet to be described. NPIF is likely to increase after decongestion, since nasal airflow is increased when measured at normal breathing ${ }^{(16)}$. However, the increase in NPIF after decongestion is limited in the presence of nasal valve stenosis ${ }^{(17)}$ and correction of nasal valve incompetence has been shown to improve nasal airflow ${ }^{(18)}$.

Describing the relationship between NPIF and NAR requires acknowledgement of three important issues. Firstly, while NAR is measured unilaterally (UNAR), conventional NPIF measures bilateral nasal airflow and changes in NPIF may be difficult to interpret in the setting of asymmetric uNAR changes. Secondly, an asymmetric response to decongestion occurs as part of the nasal cycle, with a smaller reduction in uNAR on the side with lower pre-decongestion uNAR reflecting physiological asymmetry in sympathetic tone ${ }^{(19,20)}$. Thirdly, it is important to account for the test-retest variability in rhinomanometry ${ }^{(21)}$ in defining a true change in UNAR.

The primary aim of this study was as an exploratory investigation comparing the response of NPIF to decongestion in selected populations with defined symmetrical decongestable versus structural nasal obstruction. The secondary aims were to determine if there is an ideal threshold for NPIF change for discriminating decongestable from structural nasal obstruction and to determine the accuracy of this threshold for differentiating these groups.

\section{Materials and methods}

A cross-sectional study of patients presenting with nasal obstruction at a tertiary rhinology centre was performed after obtaining approval from the institutional review board. Symptomatically-obstructed patients (rather than healthy controls) were chosen because it is known that correlation between subjective and objective measures is more likely in patients with nasal obstruction ${ }^{(22)}$. These were patients in whom the cause of nasal obstruction was not obviously rhinitis or structural based on simple history and examination. Patients with acute rhinitis, inflammatory nasal polyposis, sinonasal neoplasms, choanal atresia or stenosis, nasal septal perforation, piriform aperture stenosis and/or complete nasal obstruction were excluded. The terminology of stenosis in this study is based on the European consensus paper on rhinomanometry ${ }^{(23)}$ and refers to a fixed narrowing that does not reverse on decongestion. This is as opposed to collapse, which is a dynamic narrowing of a nasal valve on inspiration. Changes in uNAR and nasal obstruction VAS after decongestion were used to define patients into groups based on the presence or absence of decongestable nasal obstruction.

\section{Methods of assessment}

All patients had active anterior rhinomanometry and nasal obstruction VAS recorded pre and post-decongestion. Active anterior rhinomanometry was performed (GM Instruments NR6, Kilwinning, Scotland) in accordance with the 2005 consensus report of the International Rhinologic Society ${ }^{(24)}$. uNAR was calculated at $150 \mathrm{~Pa}$.

Patients scored their subjective sensation of nasal obstruction on a VAS of 0 to $100 \mathrm{~mm}(0 \mathrm{~mm}$ represented no obstruction and $100 \mathrm{~mm}$ represented complete obstruction). Each side was recorded before and after decongestion.

Decongestion was achieved by topical application of $0.05 \%$ oxymetazoline $-0.3 \mathrm{ml}$ ( 3 sprays of $0.1 \mathrm{ml}$ ) per side. All patients waited 15 minutes prior to re-testing for post-decongestion measurements.

\section{Defining a true decrease in uNAR}

A test-retest change in uNAR, which is less than the co-efficient of variation for rhinomanometry, may not represent a true difference in measurements; Silkoff reported this value to be up to $15.9 \%{ }^{(21)}$. Therefore, a 15\% decrease in uNAR after decongestion was considered the minimum to define a true decongestable change.

\section{Defining symmetry in decongestable nasal obstruction} Eccles et al. describes a strong nasal cycle both in patients with acute rhinitis and normal controls ${ }^{(19)}$. Significant asymmetry in decongestable uNAR may be a reflection of the nasal cycle rather than underlying pathology. Conventional NPIF is a measure 


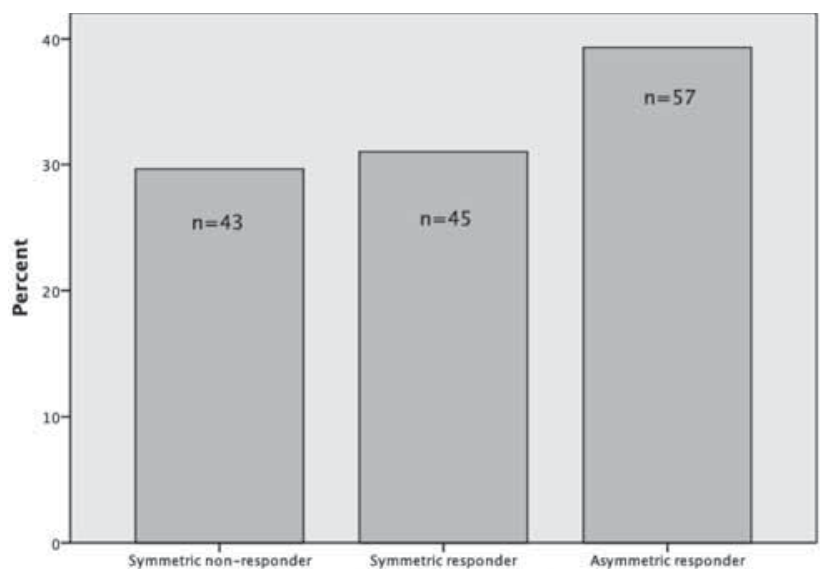

Figure 1. Symmetry of response in nasal airway resistance (NAR) to decongestion.

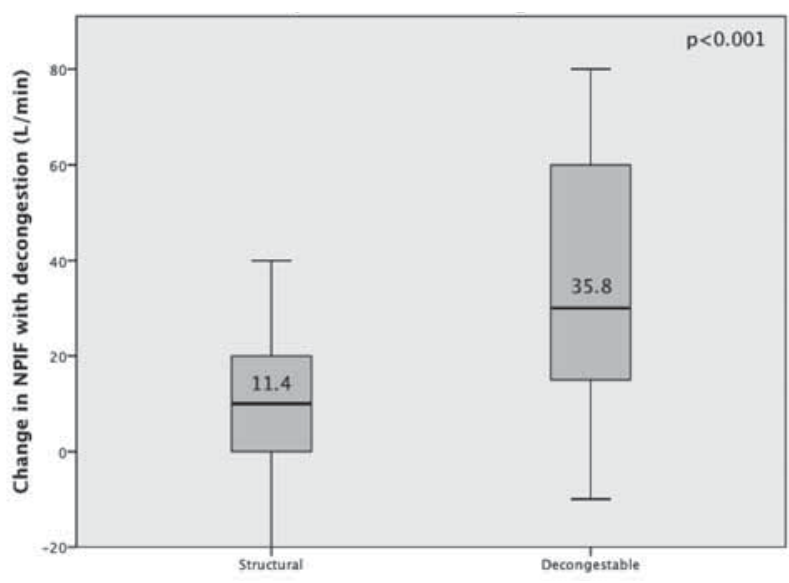

Figure 2. Comparison of change in nasal peak inspiratory flow (NPIF) (L/ min) between patients with decongestable and structural nasal obstruction. of both nasal passages together and the inclusion of patients with significant asymmetry due to the nasal cycle would make it difficult to interpret the relationship between NPIF and uNAR. For this reason, only those patients with symmetric changes were included for analysis.

Symmetrical decongestion was defined as bilateral decrease of UNAR $>15 \%$ after decongestion; bilateral lack of at least $15 \%$ decrease in uNAR or increase in uNAR defined symmetrical nondecongestable nasal obstruction. Asymmetric decongestion, was defined if the post-decongestion change between sides was $>15 \%$ on one side and $<15 \%$ on the other.

\section{Defining a true change in VAS nasal obstruction}

A true change in VAS nasal obstruction was defined as a change that was greater than the minimal clinically important difference (MCID) for nasal obstruction VAS. The distributional method of half the standard deviation was used to determine this ${ }^{(25)}$ and was calculated to be 13.0 based on standard deviation of 26.0 for the whole population.

Patients with changes in VAS nasal obstruction, which did not correspond to the changes in uNAR, were excluded as these patients are more likely to have non-anatomical factors contributing to subjective nasal obstruction ${ }^{(26)}$.

\section{Defining the study populations}

Patients with decongestable nasal obstruction were defined as having a $>15 \%$ reduction uNAR on both sides after decongestion and a decrease in nasal obstruction VAS for both sides of at least an MCID. Those with structural nasal obstruction had a reduction in post-decongestion uNAR less than $15 \%$ on both sides and no MCID improvement in nasal obstruction VAS for both sides. For the reasons explained previously, patients with asymmetric changes in uNAR or changes in nasal obstruction VAS that was inconsistent with changes in UNAR were excluded from the analysis.

\section{Outcome assessment}

NPIF (in L/min) was recorded at baseline and after decongestion. NPIF was measured in the sitting position using a mini-Wright peak flow meter with an anaesthetic mask centred over the nose and a good seal of the mask on the face. Patients were instructed to take a maximal inspiratory effort with the mouth closed. The best result of three attempts, with values within $10 \%$ of each other, was recorded ${ }^{(27,28)}$.

The change in NPIF with decongestion was derived by subtracting pre- from post-decongestion NPIF. The pre- and postdecongestion NPIF values and the change in NPIF with decongestion were compared between the 2 study populations as previously-defined.

\section{Statistical analysis}

Pre-and post-decongestion NPIF and NPIF change for each group were compared using unpaired Student's t-test. The ROC curves for the each NPIF characteristic as a predictor of decongestable or structural nasal obstruction was generated. The sensitivity, specificity, positive predictive values of NPIF change at MCID of $20 \mathrm{~L} / \mathrm{min}$ (derived from Timperley et al. ${ }^{(29)}$ and for percentage NPIF change of $>20 \%$ were calculated using a $2 \times 2$ table. Statistical analyses were performed using SPSS v 17.0 (Statistical Package for the Social Sciences, Chicago, IL, USA).

\section{Results}

\section{Characteristics of included patients}

There were 145 patients (mean age $43.5 \pm 16.5$ years, $54.7 \%$ female) in the database of patients with nasal obstruction who had presented for objective testing of the nasal airway. Of the 145 patients assessed, 57 were asymmetric responders and thus not eligible for analysis. Of the remaining 88 who had symme- 


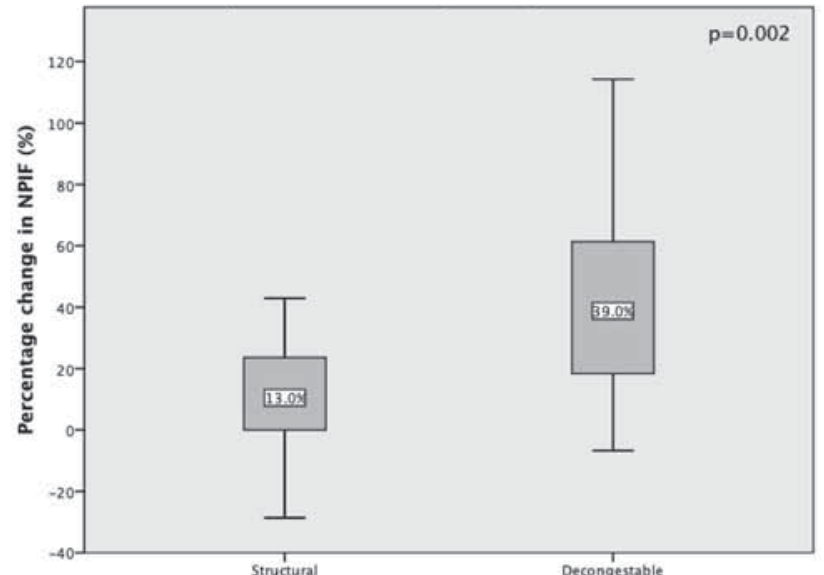

Figure 3. Comparison of percentage change in nasal peak inspiratory flow (NPIF) (L/min) between patients with decongestable and structural nasal obstruction.

trical changes in uNAR with decongestion (Figure 1), 52 (59.0\%) had VAS nasal obstruction changes, which were consistent with rhinomanometric changes. Of the final study group of $n=52$ (age $45.9 \pm 16.8$ years, $53.8 \%$ female), there were 24 patients with decongestable nasal obstruction and 28 patients with structural nasal obstruction who met the inclusion criteria of the study.

\section{Nasal peak inspiratory flow (NPIF)}

Neither pre-decongestion NPIF (105.4 $\pm 43.3 \mathrm{~L} / \mathrm{min}$ versus 107.9 $\pm 40.7 \mathrm{~L} / \mathrm{min}, \mathrm{p}=0.84)$ nor post-decongestion NPIF (141.3 \pm 50.5 $\mathrm{L} / \mathrm{min}$ versus $119.3 \pm 42.8 \mathrm{~L} / \mathrm{min}, \mathrm{p}=0.10$ ) were significantly different between decongestable and structural groups, respectively. However, mean NPIF change and mean percentage NPIF change were both larger with decongestable than structural nasal obstruction $(+35.8 \pm 25.9 \mathrm{~L} /$ min versus $+11.4 \pm 16.3 \mathrm{~L} / \mathrm{min}$ $\mathrm{p}<0.001$ ) (Figure 2 ) and (39.0 $\pm 32.6 \%$ versus $13.0 \pm 20.5 \%, \mathrm{p}=$ 0.002) (Figure 3).

To calculate the accuracy of NPIF change in predicting decongestable nasal obstruction, a minimum of $20 \mathrm{~L} / \mathrm{min}$ (i.e absolute value) improvement in NPIF was chosen as this was the reported MCID ${ }^{(29)}$. The sensitivity and specificity at this threshold were $75.0 \%$ and $60.7 \%$, respectively. The relationship was also explored for NPIF change of greater than $20 \%$ (i.e relative value), with respective sensitivity and specificity calculated at $75.0 \%$ and $64.3 \%$. The positive predictive values (PPV) were $62.1 \%$ and $64.3 \%$ for thresholds of $20 \mathrm{~L} / \mathrm{min}$ and $20 \%$ increase in NPIF, respectively. The corresponding negative predictive values (NPV) were $64.3 \%$ and $75.0 \%$. ROC analysis of the relationship between NPIF change and the presence of decongestable nasal obstruction was performed and was found to correlate $(p=0.001$ for both absolute and percentage thresholds; Figures $4 \mathrm{~A}$ and $4 \mathrm{~B}$ ). $\mathrm{A}$ change in NPIF of at least $20 \mathrm{~L} / \mathrm{min}$ was also associated with the optimum test characteristic for differentiating structural from decongestable nasal obstruction.

\section{Discussion}

There are a number of objective assessments of the nasal airway. These include rhinomanometry, acoustic rhinometry, NPIF, nasal spirometry and nasal peak expiratory flow. Characteristics of the first three tests are the most commonly-reported and their relative advantages and disadvantages have been well-described ${ }^{(3)}$.

Rhinomanometry is appropriate as a reference test in nasal obstruction. There are consistent reports of its correlation with subjective measures ${ }^{(30)}$. Unilateral sensation of nasal patency correlates well with pre-and post-decongestion NAR ${ }^{(11,31)}$.
A

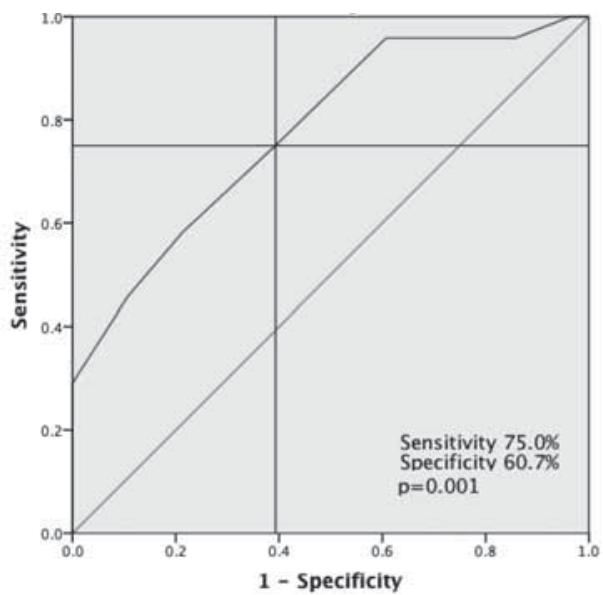

B

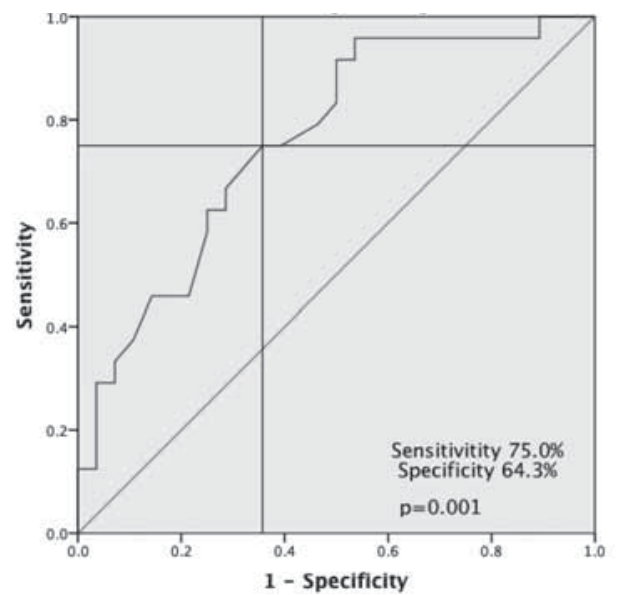

Figure 4. (A) ROC curve for NPIF change (L/min) for predicting decongestable nasal obstruction. Reference lines at threshold of $20 \mathrm{~L} / \mathrm{min}$ for NPIF change. Area under curve is 0.778 ; (B) ROC curve characteristics for percentage change in NPIF for predicting decongestable nasal obstruction. Reference lines at threshold of $20 \%$ for NPIF. Area under curve is 0.760 . 
Additionally, the more obstructed side has a greater influence on nasal obstruction VAS and when both nostrils are assessed simultaneously by patients, total NAR was found to correlate significantly with the side of greater resistance ${ }^{(13)}$. An increase in the relative difference in nasal air flow improves the ability of patients to identify the more obstructed side ${ }^{(32)}$. These features make it an essential part of objective nasal airway assessment at the tertiary level and a suitable tool to define the population groups in this study. Nevertheless, it is not easily-accessible due to its cost, time required and complexity ${ }^{(33)}$.

Measurement of NPIF may be more suitable as a screening tool as it is simple ${ }^{(34)}$, cheap, reproducible ${ }^{(27)}$ and rapid. NPIF correlates with nasal obstruction VAS ${ }^{(35)}$ and rhinoscopy ${ }^{(27)}$ and its accuracy for differentiating patients with nasal obstruction from healthy controls was reported to be similar ${ }^{(36)}$ to that of rhinomanometry.

In the present study, the change in NPIF after decongestion appears to be more valuable than the pre- or post-decongestion values of NPIF in predicting whether nasal obstruction is predominantly decongestable or structural, whereas the values of preand post-decongestion NPIF varied greatly between patients and the means were not significantly different between the study groups. This is not surprising given the wide distribution and anatomical variability that has been described ${ }^{(37,38)}$.

An optimal threshold $>20 \mathrm{~L} / \mathrm{min}$ increase in NPIF is suggested by the ROC curve for predicting decongestable change. Of note, this is similar to the MCID of NPIF as previously reported ${ }^{(29)}$. The optimum test statistic was the NPV for percentage NPIF change; the absence of at least $20 \%$ increase in NPIF was associated with a NPV of $75.0 \%$ for decongestable nasal obstruction. Such patients might have structural nasal obstruction and a more careful assessment, clinical and objective, for the presence of nasal valve stenosis or collapse should be performed prior to surgery. One possible cause of a false negative result is if inferior turbinate enlargement is primarily due to glandular hypertophy and fibrosis ${ }^{(5,39)}$, resulting in diminished reversibility with decongestion. However, NPIF is never measured in isolation and the presence of bilateral turbinate enlargement would be evident endoscopically. This may not be the case for nasal valve stenosis (which may be missed due to its very anterior position in the nasal airway) or nasal valve collapse (which is sometimes unmasked after decongestion).

It is acknowledged that the findings of this study cannot be generalized to an unselected population of patients given the strict inclusion criteria. These were chosen to minimize factors that might confound comparison between NPIF and UNAR in response to decongestion as previously explained. No form of assessment, subjective or objective, has the perfect test characteristic such that it can be used in isolation. Therefore, measuring NPIF change with decongestion is unlikely to be a replacement for more formal methods of nasal assessment. In particular, conventional NPIF cannot be applied to patients with significant asymmetry in decongestable nasal obstruction due to the nasal cycle, a problem which may be circumvented by measuring unilateral NPIF ${ }^{(40)}$. The data for this study was obtained prior to the publication of the norms for unilateral NPIF ${ }^{(40)}$. Hence, while unilateral measures would be ideal, this measurement was not available for the present group of subjects. As data on unilateral NPIF accumulates, pre- and post decongestion NPIF, measured bilaterally, may still be of value given its simplicity and availability, especially when interpreted in combination with other clinical findings.

NPIF should be part of the armamentarium in assessing nasal obstruction and an improvement in NPIF of less than $20 \mathrm{~L} / \mathrm{min}$ or $20 \%$ from baseline after decongestion should prompt the clinician to evaluate for structural causes of nasal obstruction e.g. nasal valve stenosis.

The increase in NPIF after decongestion is larger with decongestable compared with structural nasal obstruction. A lack improvement in NPIF of at least $20 \mathrm{~L} / \mathrm{min}$ or $20 \%$ may be associated with structural causes of obstruction. While this might raise suspicion of the presence of nasal valve stenosis or collapse, it is probably not recommended as a replacement for more formal assessment.

\section{Conclusion}

In conclusion, NPIF is an option for the objective evaluation of nasal airflow in septoplasty. It can also be used to assess the outcomes of inferior turbinate reduction with the aid of nasal decongestants, even in patients who also underwent septoplasty.

\section{Authorship contribution}

DC: study design, data analysis, manuscript; EP: conceptualization, study design, data collection, data analysis, manuscript edits, expert opinion; JM: data collection, data analysis, manuscript edits; GM: manuscript edits, expert opinion; RS: manuscript edits, expert opinion; KS: manuscript edits, expert opinion; RJH: conceptualization, study design, data collection, data analysis, manuscript edits, expert opinion.

\section{Acknowlegdement}

Nil funding sources to declare.

\section{Conflicts of Interest}

Richard J. Harvey, has served on an advisory board for Schering 
Plough and Glaxo-Smith-Kline, was a previous consultant with Medtronic, and serves on the speakers bureau for Merck Sharp Dohme and Arthrocare, and has received grant support from
NeilMed.

Raymond Sacks is a consultant for Medtronic and serves on the speakers bureau for Merck Sharp Dohme.

\section{References}

1. Udaka T, Suzuki H, Fujimura T et al. Chronic nasal obstruction causes daytime sleepiness and decreased quality of life even in the absence of snoring. Am J Rhinol. 2007; 21: 564-569.

2. Rhee JS, Book DT, Burzynski M, Smith TL. Quality of life assessment in nasal airway obstruction. Laryngoscope. 2003; 113:11181122.

3. Chandra RK, Patadia MO, Raviv J. Diagnosis of nasal airway obstruction. Otolaryngol Clin North Am. 2009; 42: 207-225, vii.

4. Nurse LA, Duncavage JA. Surgery of the inferior and middle turbinates. Otolaryngol Clin North Am. 2009; 42: 295-309, ix.

5. Berger G, Gass S, Ophir D. The histopathology of the hypertrophic inferior turbinate. Arch Otolaryngol Head Neck Surg. 2006; 132: 588-594.

6. Becker SS, Dobratz EJ, Stowell N, Barker D, Park SS. Revision septoplasty: review of sources of persistent nasal obstruction. Am J Rhinol. 2008; 22: 440-444.

7. Sulsenti G, Palma P. Tailored nasal surgery for normalization of nasal resistance. Facial Plast Surg. 1996; 12: 333-345.

8. Sulsenti G, Palma P. A new technique for functional surgery of the nasal valve area. Rhinol Suppl. 1989; 10: 1-19.

9. Sulsenti G, Palma P. [The nasal valve area: structure, function, clinical aspects and treatment. Sulsenti's technic for correction of valve deformities]. Acta Otorhinolaryngol Ital. 1989; 9 Suppl 22: 1-25.

10. Szucs E, Clement PA. Acoustic rhinometry and rhinomanometry in the evaluation of nasal patency of patients with nasal septal deviation. Am J Rhinol. 1998; 12: 345-352.

11. Roithmann R, Cole P, Chapnik J, Barreto SM, Szalai JP, Zamel N. Acoustic rhinometry, rhinomanometry, and the sensation of nasal patency: a correlative study. J Otolaryngol 1994; 23: 454-458.

12. Thulesius HL, Cervin A, Jessen M. The importance of side difference in nasal obstruction and rhinomanometry: a retrospective correlation of symptoms and rhinomanometry in 1000 patients. Clin Otolaryngol. 2012; 37: 17-22.

13. Tompos T, Garai T, Zemplen B, Gerlinger I. Sensation of nasal patency compared to rhinomanometric results after septoplasty. Eur Arch Otorhinolaryngol. 2010; 267: 18871891.

14. Sanz L, Guerrero JA, Rivera T. [Correlation between active anterior rhinomanometry and nasal endoscopy]. Acta Otorrinolaringol Esp. 2010; 61:215-219.

15. Ciprandi G, Cirillo I, Vizzaccaro A, Pallestrini
E, Tosca MA. Decongestion test in patients with allergic rhinitis: functional evaluation of nasal airflow. Am J Rhinol. 2006; 20: 224226.

16. Ciprandi G, Klersy C, Ameli F, Cirillo I. Clinical assessment of a nasal decongestion test by visual analog scale in allergic rhinitis. Am J Rhinol. 2008; 22: 502-505.

17. Shaida AM, Kenyon GS. The nasal valves: changes in anatomy and physiology in normal subjects. Rhinology. 2000; 38: 7-12.

18. Constantian MB, Clardy RB. The relative importance of septal and nasal valvular surgery in correcting airway obstruction in primary and secondary rhinoplasty. Plast Reconstr Surg. 1996; 98: 38-54.

19. Williams RG, Eccles R. Nasal airflow asymmetry and the effects of a topical nasal decongestant. Rhinology. 1992; 30: 277-282.

20. Flanagan P, Eccles R. Physiological versus pharmacological decongestion of the nose in healthy human subjects. Acta Otolaryngologica. 1998; 118: 110-113.

21. Silkoff PE, Chakravorty S, Chapnik J, Cole P, Zamel N. Reproducibility of acoustic rhinometry and rhinomanometry in normal subjects. Am J Rhinol. 1999; 13: 131-135.

22. Andre RF, Vuyk HD, Ahmed A, Graamans K, Nolst Trenite GJ. Correlation between subjective and objective evaluation of the nasal airway. A systematic review of the highest level of evidence. Clin Otolaryngol. 2009: 34: 518-525.

23. Vogt K, Jalowayski AA, Althaus Wet al. 4-Phase-Rhinomanometry (4PR)--basics and practice 2010. Rhinol Suppl. 2010: 1-50.

24. Clement PA, Gordts F. Consensus report on acoustic rhinometry and rhinomanometry. Rhinology. 2005; 43: 169-179.

25. Wyrwich KW, Tierney WM, Wolinsky FD. Further evidence supporting an SEM-based criterion for identifying meaningful intraindividual changes in health-related quality of life. J Clin Epidemiol. 1999; 52: 861-873.

26. Hopkins C. Re: Correlation between subjective and objective evaluation of the nasal airway. Clin Otolaryngol. 2010; 35: 147-148; author reply 148.

27. Starling-Schwanz R, Peake HL, Salome CM, et al. Repeatability of peak nasal inspiratory flow measurements and utility for assessing the severity of rhinitis. Allergy. 2005; 60: 795-800.

28. Jones AS, Viani L, Phillips D, Charters P. The objective assessment of nasal patency. Clin Otolaryngol Allied Sci. 1991; 16: 206-211.

29. Timperley D, Srubisky A, Stow N, Marcells GN, Harvey RJ. Minimal clinically important differences in nasal peak inspiratory flow. Rhinology. 2011; 49: 37-40.
30. Mora F, Cassano M, Mora R, Gallina AM, Ciprandi G. V.A.S. in the follow-up of turbinectomy. Rhinology. 2009; 47: 450-453.

31. Hirschberg A, Rezek O. Correlation between objective and subjective assessments of nasal patency. ORL J Otorhinolaryngol Relat Spec. 1998; 60: 206-211.

32. Clarke JD, Hopkins ML, Eccles R. How good are patients at determining which side of the nose is more obstructed? A study on the limits of discrimination of the subjective assessment of unilateral nasal obstruction. Am J Rhinol. 2006; 20: 20-24.

33. Holmstrom M. The use of objective measures in selecting patients for septal surgery. Rhinology. 2010; 48: 387-393.

34. Dufour X, Gohler C, Delagranda A, Fontanel JP, Klossek JM. [Peak Nasal Inspiratory Flow: learning curve for the measurement method and reproducibility]. Ann Otolaryngol Chir Cervicofac. 2007; 124: 115-119.

35. Kjaergaard T, Cvancarova M, Steinsvag SK. Does nasal obstruction mean that the nose is obstructed? Laryngoscope. 2008; 118: 1476-1481.

36. Bermuller $C$, Kirsche $H$, Rettinger $G$, Riechelmann H. Diagnostic accuracy of peak nasal inspiratory flow and rhinomanometry in functional rhinosurgery. Laryngoscope 2008; 118: 605-610.

37. Blomgren K, Simola M, Hytonen $M$, Pitkaranta A. Peak nasal inspiratory and expiratory flow measurements--practical tools in primary care? Rhinology 2003; 41: 206-210.

38. Ottaviano G, Scadding GK, Coles S, Lund VJ. Peak nasal inspiratory flow; normal range in adult population. Rhinology. 2006; 44: 32-35.

39. Schmidt J, Zalewski P, Olszewski J, Olszewska-Ziaber A. Histopathological verification of clinical indications to partial inferior turbinectomy. Rhinology. 2001; 39: 147-150.

40. Ottaviano G, Scadding GK, Scarpa B, Accordi D, Staffieri A, Lund VJ. Unilateral peak nasal inspiratory flow, normal values in adult population. Rhinology. 2012; 50: 386-392.

\section{Dr David Chin}

354 Victoria Street

Darlinghurst

NSW 2010

Australia 